\section{Aus den Ländern Pflegekammer NRW: Es geht voran}

Der Fokus der Arbeit des BLGS-Landesverbands NRW liegt weiterhin auf der Errichtung einer Pflegekammer für NRW. In Zusammenarbeit mit dem Landespflegerat startet der BLGS eine Initiative mit dem Ziel, die Mitglieder der Pflegeberufe über die Pflegekammer zu informieren. Diesbezüglich ist eine Master-Präsentation zur Etablierung einer Pflegekammer für NRW entstanden, die von verschiedenen Multiplikatoren in Einrichtungen der Pflege vorgestellt werden soll. Ein weiterer Schwerpunkt dieser Initiative ist der Austausch mit den Parteien des Landtags; hier konnte erwirkt werden, dass die CDU-Fraktion das Anliegen des BLGS und des Landespflegerats (www.pflegerat-nrw.de) unterstützt. Im Oktober soll hierzu eine Verbändeanhörung stattfinden. Natürlich wird der BLGS Präsenz zeigen und seine Position vertreten.

Wie bereits berichtet, wurde für die Qualitätssicherung der Ausbildung in der Altenpflege eine Arbeitsgruppe vom Ministerium für Gesundheit, Emanzipation, Pflege und Alter einberufen, um Qualitätsstandards zu definieren. Unter anderem sprach sich die Arbeitsgruppe für ein Lehrer-Schüler-Verhältnis von 1:50 aus. Der BLGS NRW kritisiert dieses Verhältnis als völlig unzureichend und zog sich daher aus der Arbeitsgruppe zurück.

Im Rahmen der neu geschaffenen Internetpräsenz https:// www.pflegeberufe-nrw.de hat der BLGS NRW finanzielle Unterstützung bei der Erstellung eines Imagevideos für den ambulanten Bereich der Altenpflege geleistet (https://www.pflegeberufe-nrw. de/berufe/altenpflege).

Besonders freuen wir uns auf unsere diesjährige Fachtagung am 27. Oktober in den Westfalenhallen Dortmund. In diesem Jahr wird es vorrangig um die Aufgaben, Möglichkeiten und Grenzen der Praxisanleitung gehen, wozu wir u.a. Dr. German Quernheim als Referenten gewinnen konnten.

Daniela Schlosser

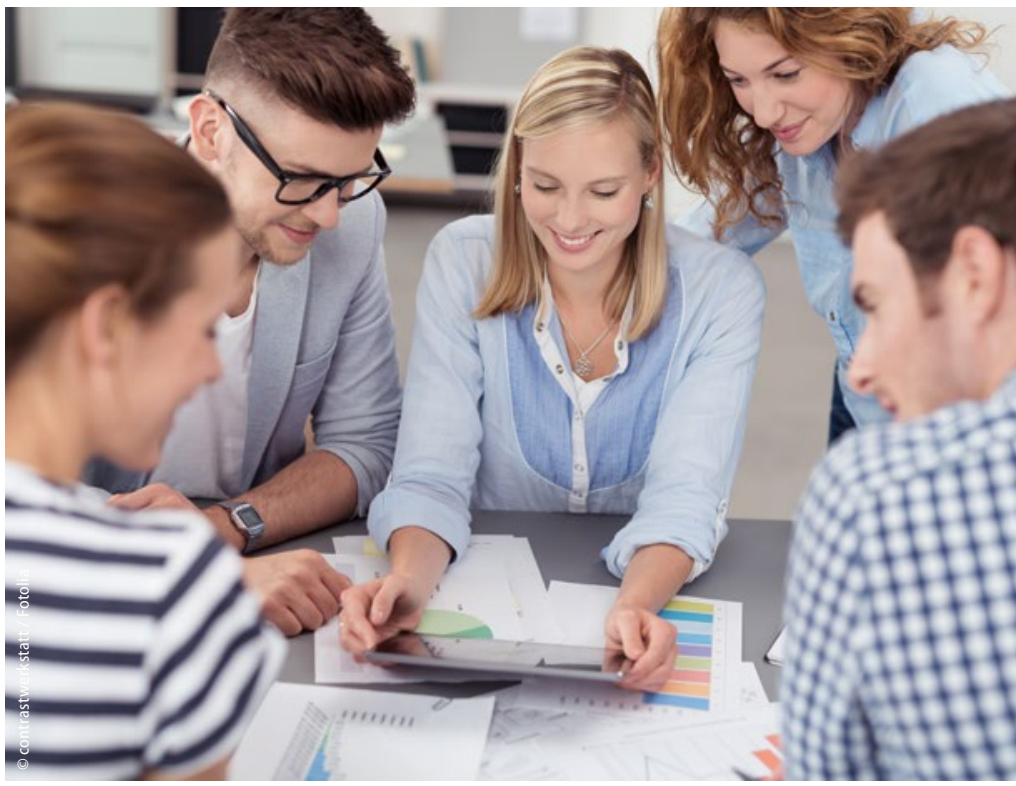

Der BLGS NRW hat sich für die Erstellung eines Imagevideos für die ambulante Altenpflege stark gemacht.

\title{
LV Berlin: Erste Landesversammlung mit Fachtagung
}

Am 23. Februar 2016 fand in Berlin die 1. Landesversammlung mit Fachtagung des BLGS LV Berlin in den Räumen der WannseeSchule e. V. statt. Es war die erste Landesversammlung nach dem Übertritt der LAG Berlin in den Bundesverband. Themen waren neben dem Geschäfts- und Haushaltsbericht des Vorstands die Gründung einer "Fachgruppe Praxis". Anke Jakobs, Mitglied im Landesvorstand, informierte über die geplante Plattform für Praxisanleiter im Landesverband. Damit soll den Praxisanleitern der Stadt ein Forum angeboten werden, um die Praxisanleitung als eine zentrale Aufgabe zu stärken und unter den aktuellen und zukünftigen Bedingungen aktiv zu gestalten. Der LV Berlin lädt als Auftaktveranstaltung zu einem Praxisanleiter-Symposium am 11.10.2016 ein (www.blgs-Iv-berlin.de). Des Weiteren informierte Landesvorsitzende Christine Vogler über den aktuellen Stand des neuen Pflegeberufsgesetzes.

Im Anschluss an die Landesversammlung fand für Mitglieder und Gäste eine Fachtagung mit zwei Vorträgen statt:

Prof. Dr. Doris Schaeffer referierte zum Thema "Interprofessionalität als Voraussetzung für bedarfsgerechte Versorgung". Angesichts der steigenden Herausforderungen müsse eine neue Kultur der vernetzten Zusammenarbeit der Gesundheitsberufe etabliert werden. Frau Schaeffer plädierte für innovative interprofessionelle
Versorgungsmodelle, die auch eine Veränderung der Bildung voraussetzen: Interprofessionalität und Kooperation wollen gelernt sein, deshalb müssen Programme zu interprofessionellem Lernen geschaffen werden.

Prof. Dr. Barbara Knigge-Demal referierte zum Thema „Qualifikationsrahmen und Kompetenzentwicklung im Curriculum". Sie stellte die Ziele des Europäischen Qualifikationsrahmens (EQR) dar, aber auch dessen Grenzen: Er will nicht nationale/sektorale/ domänenspezifische oder Fachqualifikationsrahmen ersetzen, er will keine Anpassung der europäischen Länder, er definiert keine neuen Qualifikationen/Abschlüsse und ist nicht personenbezogen. Eindrucksvoll präsentierte sie mit verschiedenen Beispielen Pflegebildungsinhalte auf unterschiedlichem Qualifikationsniveau im Deutschen Qualifikationsrahmen (DQR). Deutlich wurde auch, dass die im Entwurf des neuen Pflegeberufsgesetzes formulierte Vorbehaltsaufgabe besondere Auswirkungen auf die Ausbildung und die Kompetenzen der beruflich Pflegenden haben wird.

Wir haben die nächste Landesversammlung mit Fachtagung für den 22.02.2017 geplant und laden Mitglieder und Interessierte schon heute herzlich dazu ein! 